“C 2010 IEEE. Personal use of this material is permitted. Permission from IEEE must be obtained for all other uses, in any current or future media, including reprinting/republishing this material for advertising or promotional purposes, creating new collective works, for resale or redistribution to servers or lists, or reuse of any copyrighted component of this work in other works.” 


\title{
Steady and Dynamic Performance Analyses of a Linear Induction Machine
}

\author{
Wei Xu, Jianguo Zhu, Yongchang Zhang, Youguang Guo, Guangyong Sun
}

\begin{abstract}
This paper based on the winding function algorithm presents an improved equivalent circuit to analyze single linear induction motors (SLIMs) applied in the linear metro. The circuit deduced from the air-gap magnetic flux density equations can analyze steady and transient performances considering end effects, half filled slots, saturated iron and skin effect. Firstly several stable cases like constant thrust/power region, constant current constant frequency or variable frequencies, constant voltage constant frequency or variable frequencies are presented in detail. Then, maximal thrust at a given speed is optimized by modifying the optimal slip frequency. Finally, transient characteristics of SLIM under constant current constant frequency are investigated. The results indicate that winding function method is one effective way to study SLIM, especially its end effects. It can be used in the electromagnetic design and performance investigation for SLIM combining relative control schemes.
\end{abstract}

Index Terms-Single-sided linear induction machine (SLIM); longitudinal end effect; transversal edge effect; winding function method; linear metro; performance analysis; equivalent circuit.

\section{INTRODUCTION}

$\mathrm{T}$ HE linear induction motors (LIMs) have been used for many industrial applications, for shuttle and medium speed transportation drive systems, such as propulsion system in the high speed surface transport (HSST) developed by Japan, and the linear metro exploited by Bombardier company in Canada [1-5]. Some advantages of using an LIM for vehicle propulsion are direct thrust achieved without reliance on friction between wheel and rail, great hill-climbing ability, and reduced cross-sectional area without large volume of gear boxes. The simple diagram of linear metro propelled by LIM is shown in Fig. 1. Comparing to rotary induction motor (RIM) drive system, the primary from stator is placed on the vehicle, while the secondary from rotor is fixed in the rail track [6-10].

From Fig. 1, the primary of single-sided LIM (SLIM) is nearly the same as that of RIM. When balanced three-phase currents flow in the windings, a spatially sinusoidal magnetomotive force is produced. For the SLIM is conceptually from cut-open RIM, a problem of arranging the conductors near both terminals in primary will occur when a double-layer winding is adopted. This phenomenon is half-filled slot that could reduce the average flux linkage in the air gap. The SLIM secondary usually consists of a sheet rail with laminated back iron that has great robustness

Manuscript received May 12, 2010; revised June 15, 2010.

W. Xu, J. G. Zhu, Y. C. Zhang, Y. G. Guo are with School of Electrical, Mechanical and Mechatronic Systems, University of Technology, Sydney, Australia (e-mail: weixuhappy@ieee.org).

G.Y. Sun is with the State Key Laboratory of Advanced Design and Manufacture for Vehicle Body, Hunan University, Changsha, China. suitable to the transportation. By plenty of investigation, the greatest thrust could be obtained with an aluminum-capped rail together with high power factor and efficiency [4], which is the structure discussed in this paper.

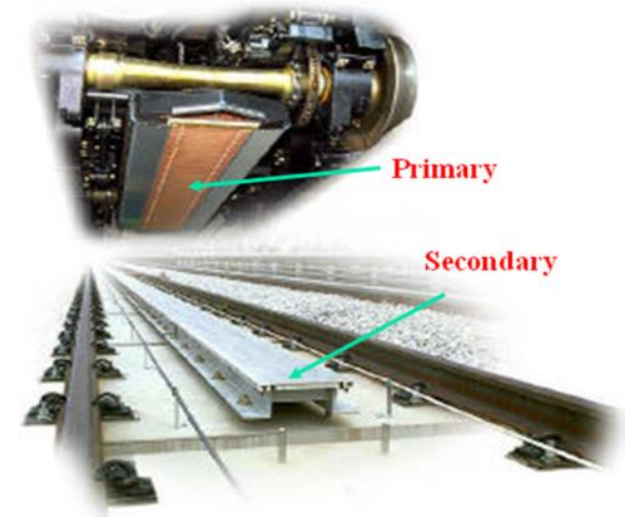

Fig. 1. Simple diagram of linear metro driven by SLIM.

Some papers are available on the analysis of SLIM performance. An equivalent circuit is one effective method. Ref. [1] has proposed a T-model equivalent circuit and corresponding correction coefficients for end effects, which could be applied in wide range. However, it is not suitable to control and dynamic analysis for the whole derivation is based on the steady-state equations. Refs. [2] and [11] have gained one function according to secondary eddy current average and conversion energy balance, which is affected by speed and structure parameters. This method can be applied easily in SLIM control schemes. However, this algorithm is very coarse, whose error would increase as the velocity goes up. Ref. [3] can theoretically simulate stable and dynamic performance by space harmonic method, but it requires more substantial computing time to gain the useful results. The precision of final results is closely relative with the initial evaluation. If some deviations from actual values exit at the beginning of solution, great error might occur in the end. Some experts have developed coupled-circuit methods to investigate the SLIM steady-state and dynamic (transient) performance, such as Elliott, Ooi, North and Lipo. In all the methods, it is supposed that end effects do not allow the use of modeling similar to that for an RIM. As we know, the RIM behavior needs to be computed only over one pole pitch, and then the whole performance over the remaining pole pitches could be obtained only by using the property of symmetry. However, symmetry arguments are not suitable to SLIM, because some severe electromagnetic conditions could change both at entry and exit ends.

This paper sets out an improved model, which can be used conveniently to analyze steady and transient performances of SLIM. The steady performance includes all kinds of behaviors in steady voltage or current excitations, and transient performance includes electromagnetic 
transitional behavior. Winding function method is also used to calculate circuit parameters, such as mutual inductance and secondary resistance.

\section{EQuivalent CiRcuit Model of SLIM}

In order to simplify the whole derivation, we firstly suppose the following assumptions $[1,8,10]$ :

(a) The stator iron has infinite permeability.

(b) All magnetic variables are sinusoidal time functions.

(c) Winding space harmonics are negligible.

(d)The primary and secondary currents flow in infinitesimally thin sheets.

(e) Three-phase currents in the primary are balanced.

On the base of air-gap flux density equation, the paper divides secondary winding function of SLIM into fundamental component and end effect component. To begin with, it deduces the primary two-phase stationary axis according to the primary winding distribution. The secondary fundamental one and end effect one are gained separately from stable state and dynamic state of gap magnetic flux equations. Moreover, it calculates all inductances, goodness factor, secondary resistance and speed voltage coefficients, then achieves voltage and flux equations. On the energy conversion relationship between primary and secondary, it gets the expressions of thrust, power factor and efficiency. In general, the whole derivation progress is a little complex, more details are shown in $[4,8]$. A summary of this model is presented below.

From Ampere's law, Faraday's law and Maxwell's law, the air-gap flux density equation of SLIM can be expressed by [4]

$$
\frac{\partial^{2} b_{y}}{\partial x^{2}}-\sigma \mu_{0} v_{2} \frac{\partial b_{y}}{\partial x}-j \sigma \mu_{0} \omega_{e} b_{y}=-j \mu_{0} \frac{\pi}{\tau} J_{1} e^{j\left(\omega_{e} t-\pi x / \tau\right)}
$$

where $\sigma$ is the secondary conductivity, $\mu_{0}$ the air permeability, $v_{2}$ the primary operating speed, $\omega_{\mathrm{e}}$ the angular frequency of primary power supply, $\tau$ the primary pole pitch, $J_{1}$ the equivalent primary sheet current density, and $b_{\mathrm{y}}$ the flux density in the $y$-axis direction. The solution of $b_{\mathrm{y}}(x, t)$ is

$$
b_{y}(x, t)=\dot{B}_{0} e^{-j \frac{\pi x}{\tau}}+\dot{B}_{1} e^{-\frac{x}{\alpha_{1}}} e^{-j \frac{\pi x}{\tau_{e}}}+\dot{B}_{2} e^{\frac{x}{\alpha_{2}}} e^{j \frac{\pi x}{\tau_{e}}}
$$

where $b_{\mathrm{y}}$ consists of three parts, $B_{0}, B_{1}$ and $B_{2} . B_{0}$ is the normal traveling wave which travels forward like the fundamental flux density in the RIM. $B_{1}$ and $B_{2}$, determined from boundary conditions, are the entrance and exit end-effect waves, respectively. $\alpha_{1}$ is the penetration depth of entry-end-effect wave, $\alpha_{2}$ the penetration depth of exit-end-effect wave, and $\tau_{\mathrm{e}}$ the half wavelength of end effect wave, which are functions of speed and motor structural parameters [8].

On the base of air-gap flux density equation, this paper divides SLIM secondary winding function into fundamental and end effect components. Firstly it deduces the primary two-phase stationary axis model according to the primary winding distribution. Then the secondary fundamental and end effect parts are gained separately from the steady state and dynamic state of air gap magnetic flux equations. Moreover, it calculates all inductances, goodness factor, secondary resistance and speed voltage coefficients. On the energy conversion relationship between primary and secondary, the mathematic expressions of thrust, power factor and efficiency are derived. Generally, the whole derivation progress is complex, and more details can be found in $[4,8]$. A brief summary is made below.

The primary stationary three-axis winding functions are

$$
\left\{\begin{array}{l}
N_{a s}(x)=\frac{N_{s}}{2} \cos (\pi x / \tau+\pi) \\
N_{b s}(x)=\frac{N_{s}}{2} \cos (\pi x / \tau+\pi / 3) \\
N_{c s}(x)=\frac{N_{s}}{2} \cos (\pi x / \tau-\pi / 3)
\end{array}\right.
$$

where $N_{\mathrm{s}}$ is the fundamental part per pole winding series.

In order to gain the stationary two-axis stator winding function expressions, $N_{\alpha \mathrm{s}}$ and $N_{\beta \mathrm{s}}$, from the stationary three-axis winding distributions, the following rules according to the flux linkage balance theory should be obeyed

$$
N_{a s}(x) i_{a s}+N_{b s}(x) i_{b s}+N_{c s}(x) i_{c s}=N_{\alpha s}(x) i_{\alpha s}+N_{\beta s}(x) i_{\beta s}
$$

where $i_{\text {as }}$ and $i_{\beta \mathrm{s}}$ can be achieved from $i_{\mathrm{as}}, i_{\mathrm{bs}}$ and $i_{\mathrm{cs}}$ by using static $3 / 2$ coordination transformation. Hence, the primary stationary two-axis winding functions are expressed by

$$
\left\{\begin{array}{l}
N_{\alpha s}(x)=\frac{3}{4} N_{s} \sin (\pi x / \tau) \\
N_{\beta s}(x)=-\frac{3}{4} N_{s} \cos (\pi x / \tau)
\end{array}\right.
$$

The secondary winding functions including both the fundamental and end effect parts are abstract, which can be gained from the electromagnetic relationship between primary and secondary $[1,8]$. The secondary fundamental winding function $\dot{N}_{r s}$ is derived by

$$
\dot{B_{r s}}=\frac{\mu_{0}}{g_{e}} \dot{N}_{r s} \dot{I}_{r s}
$$

where $\dot{B}_{r s}, \dot{I}_{r s}$ and $g_{\mathrm{e}}$ are the secondary fundamental complex flux density, secondary fundamental complex current and equivalent electromagnetic air gap width respectively. From the air gap flux density steady equation, $\dot{B_{r s}}$ can be expressed by

$$
\dot{B}_{r s}=\frac{\left(3 \mu_{0} / 4 g_{e}\right) N_{s}}{\sqrt{1+(1 / s G)^{2}}} I_{s} \exp \left[j\left(\omega_{e} t-\pi x / \tau+\theta_{s}\right)\right]
$$

where $s$ is the slip, $G$ the goodness factor, $\theta_{\mathrm{s}}$ the angle between the primary and secondary fundamental currents, and $I_{\mathrm{s}}$ the RMS value of primary phase current. From the T-model equivalent circuit of one dimension, $\dot{I}_{r s}$ can be drawn by

$$
\dot{I}_{r s}=\dot{I_{s}} \frac{-j X_{m}}{j X_{m}+\frac{R_{r}}{s}}=\frac{-I_{s}}{\sqrt{1+(1 / s G)^{2}}} \exp \left[j\left(\omega_{e} t+\theta_{s}\right)\right]
$$

where $X_{\mathrm{m}}$ and $R_{\mathrm{r}}$ are the mutual inductance and secondary resistance. Hence, from (6) to (8), the secondary 
fundamental winding functions $\dot{N}_{r s}$ are indicated by

$$
\left\{\begin{array}{l}
N_{\alpha r s}(x)=\frac{3}{4} N_{s} \sin (\pi x / \tau) \\
N_{\beta r s}(x)=-\frac{3}{4} N_{s} \cos (\pi x / \tau)
\end{array}\right.
$$

Similar with the solution of secondary fundamental part, the secondary end effect part winding function $\dot{N}_{r e}$ can be deduced by

$$
\dot{B}_{r e}=\frac{\mu_{0}}{g_{e}} \dot{N}_{r e} \dot{I}_{r e}
$$

where $\dot{B_{r e}}$ and $\dot{I}_{r e}$ are the secondary end effect complex flux density and secondary end effect complex current, respectively. From the air gap flux density dynamic equation, $\dot{B}_{r e}$ can be derived by

$$
\dot{B}_{r e}=\frac{\frac{3 \mu_{0}}{4 g_{e}} N_{s} I_{s}\left(\frac{1}{\alpha_{2}}+s G \frac{\pi}{\tau}+j \frac{\pi}{\tau_{e}}\right)}{\left(\frac{1}{\alpha_{1}}+\frac{1}{\alpha_{2}}+j \frac{2 \pi}{\tau_{e}}\right)(1+j s G)} \exp \left(-x / \alpha_{1}\right) \exp \left[j\left(\omega_{e} t-\frac{\pi x}{\tau_{e}}\right)\right]
$$

$$
\text { From }[5], \quad \dot{I}_{r e} \text { is }
$$

$$
\dot{I}_{r e}=K \frac{1}{1+j s G} I_{s} \exp \left(j \omega_{e} t\right)
$$

where $K$ is a function of the SLIM velocity and primary length, etc. From (10) to (12), the secondary end effect winding function $\dot{N}_{r e}$ is shown as

$$
\left\{\begin{array}{l}
N_{\alpha r e}(x)=-\frac{N_{2}}{K} \exp \left(-x / \alpha_{2}\right) \sin \left(\pi x / \tau_{e}-\theta_{e}\right) \\
N_{\beta r e}(x)=\frac{N_{2}}{K} \exp \left(-x / \alpha_{2}\right) \cos \left(\pi x / \tau_{e}-\theta_{e}\right)
\end{array}\right.
$$

where $N_{2}$ and $\theta_{\mathrm{e}}$ are related with the slip, goodness factor and SLIM structural parameters.

According to aforementioned three groups of winding function equations, their profiles are indicated in Fig. 2 based on a 6-pole SLIM. It is interesting to see that the primary two-axis parts are the same as the secondary fundamental ones, which are sinusoidal waves within the primary length. For the influence of primary half-filled slots, the $\beta$-axis wave can be regarded as lagging the $\alpha$-axis one by $\pi / 2$. The secondary end effect parts attenuate gradually from entrance side to exit side for the longitudinal end effect influence.

The SLIM voltage equation in matrix form is given by

$$
\vec{u}=[R] \vec{i}+\frac{d \vec{\lambda}}{d t}+v_{2} \frac{\pi}{\tau}[U] \vec{\lambda}
$$

where several vectors are expressed by the following.

$$
\vec{\lambda}=[L] \vec{i}, \vec{u}=\left[u_{\alpha s}, u_{\beta s}, 0,0\right]^{T}, \vec{i}=\left[i_{\alpha s}, i_{\beta s}, i_{\alpha r}, i_{\beta r}\right]^{T} \text {, }
$$

$[R]=\left[\begin{array}{cccc}R_{s} & 0 & 0 & 0 \\ 0 & R_{s} & 0 & 0 \\ 0 & 0 & R_{r} & 0 \\ 0 & 0 & 0 & R_{r}\end{array}\right],[U]=\left[\begin{array}{cccc}0 & 0 & 0 & 0 \\ 0 & 0 & 0 & 0 \\ 0 & 0 & 0 & 1 \\ 0 & 0 & -1 & 0\end{array}\right]$.

where $L$ is an inductance matrix, in which the element between any two winding functions mentioned in (5), (9), (13) may be calculated by

$$
L_{12}=\frac{2 \mu_{0} l_{\delta}}{3 g_{e}} \int_{0}^{p \tau} N_{1}(x) N_{2}(x) d x
$$

where $N_{1}(\mathrm{x})$ and $N_{2}(\mathrm{x})$ are two arbitrary winding functions, $l_{\delta}$ is the primary stack width, and $p$ is number of primary poles. There are 36 inductances to be calculated, where 18 of them are independent [4].

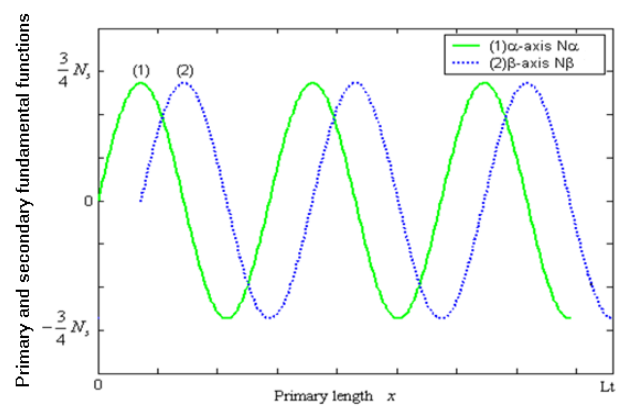

(a)

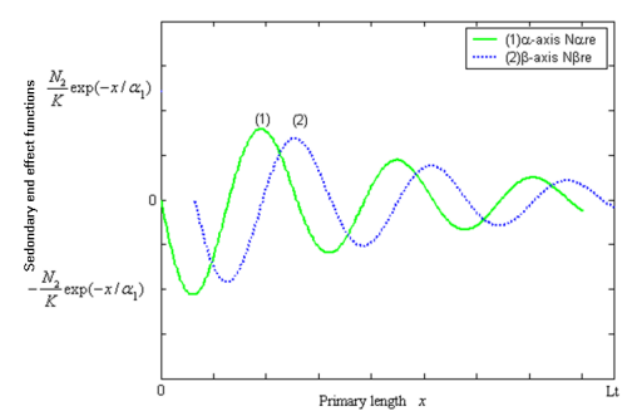

(b)

Fig. 2. Profiles of different winding functions: (a) primary and secondary fundamental parts, (b) secondary end effect parts.

According to the energy conversion balance theorem between primary and secondary, the thrust is

$$
F_{e}=(3 \pi / 2 \tau)\left[i_{1}\right]^{T}[G]\left[i_{1}\right]
$$

where matrix $i_{1}$ including the end effect part is expressed by

$$
\left[i_{1}\right]=\left[i_{\alpha s}, i_{\beta s}, i_{\alpha r}, i_{\beta r},\left(i_{\alpha s}+i_{\alpha r}\right),\left(i_{\beta s}+i_{\beta r}\right)\right]^{T}
$$

and matrix $G$ is the speed voltage coefficient matrix, which may be calculated in the similar way of inductance matrix $L$.

The input active power is

$$
p_{i n}=\frac{3}{2}\left(u_{\alpha s} i_{\alpha s}+u_{\beta s} i_{\beta s}\right)
$$

The input reactive power is

$$
q_{\text {in }}=\frac{3}{2}\left(u_{\beta s} i_{\alpha s}-u_{\alpha s} i_{\beta s}\right)
$$

The output active power is

$$
p_{\text {out }}=\left(F_{e}-F_{m}\right) v_{2}
$$


where $F_{\mathrm{m}}$ is the total mechanical resistant force involving wind and friction forces.

The power factor is

$$
\cos \varphi=p_{\text {in }} / \sqrt{{p_{\text {in }}{ }^{2}+q_{\text {in }}{ }^{2}}^{2}}
$$

The efficiency is

$$
\eta=p_{\text {out }} / p_{\text {in }}
$$

\section{SIMULATION AND EXPERIMENTATION}

The above derivations describe transient state of SLIM in differential form of linkage. The equations could be solved by numerical step by step method. In most dynamic cases, the state variables include secondary linkages and primary currents. The equations can also be readily used to steady-state analysis, which can be calculated by simply setting $d / d t$ to $j \omega$ and solving (14). This paper has studied steady and dynamic states thrust curves of high power SLIM under current control or voltage control. The main parameters are shown in table I.

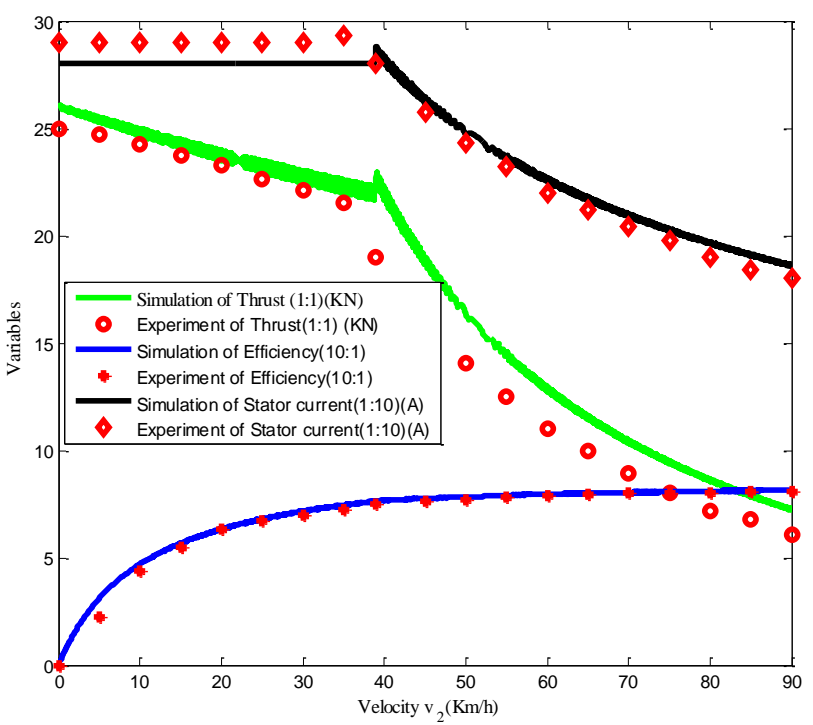

Fig. 3. Primary phase current, thrust, and efficiency variable curves.

Fig. 3 has analyzed the SLIM steady-state drive performance in the overall working region and compared simulations with measurements including the phase current, thrust and efficiency curves [9]. $40 \mathrm{~km} / \mathrm{h}$ is chosen as the base speed according the practical requirement. Simulation evaluations on the phase current, thrust, and efficiency are made according to the steady equations of the winding function equations. The experimental setup and measurement values are referred in [3]. Simulated phase current is close to its measurement, which is kept constant below base speed and decreases linearly beyond that for its limitable maximal phase voltage. The thrust below the base speed decreases a little as the speed goes up for the end effect although the phase current is constant. Beyond the base point, thrust decreases linearly for the reducing phase current. Error in the base speed is obvious because the control manner and slip frequency have been changed greatly. The efficiency calculation and measurement agree with each other approximately, which meet engineering application requirement.

Fig. 4 is the thrust curves calculated by stable state equations whose primary current is kept constant, $280 \mathrm{~A}$. Dash lines indicate thrusts without considering end effects, but the continuous lines consider end effects. Due to larger air gap and end effects, the thrust should decrease as velocity goes up [5]. Fig. 5 is the thrust curves calculated by stable state equations whose primary phase voltage is kept constant, $635 \mathrm{~V}$. The trend of thrust is similar to that of Fig. 4. For the quick attenuation of the phase current, the thrust in constant voltage decreases more quickly for the increasing total impedance than that of constant current.

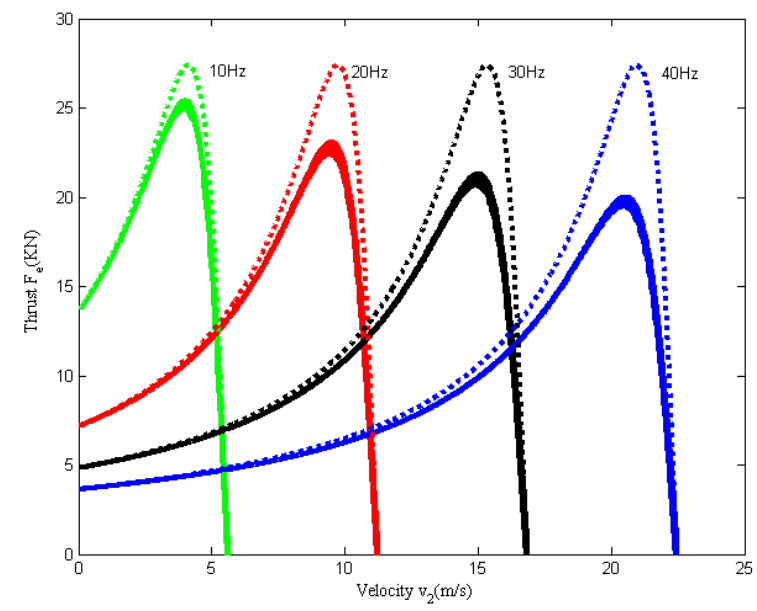

Fig. 4. Thrust curves with constant current variable frequency, calculated by stable state

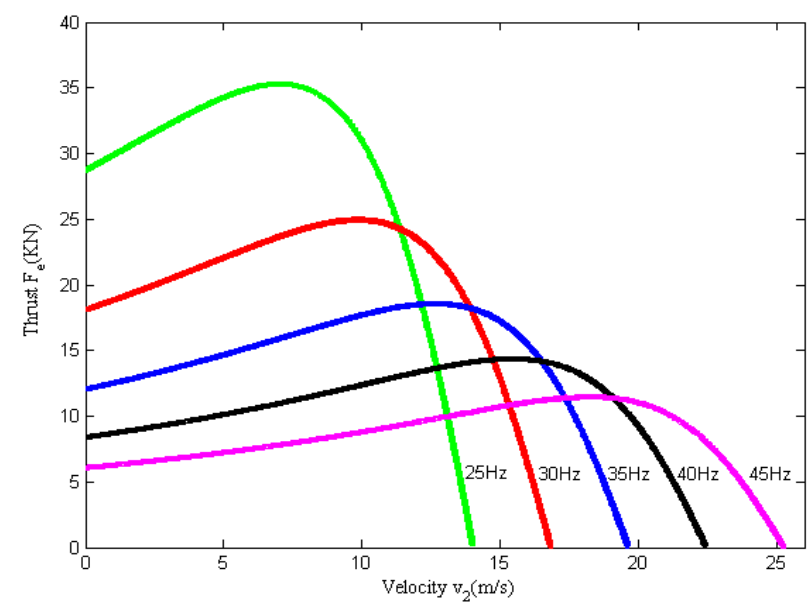

Fig. 5. Thrust curves with constant voltage variablefrequency, calculated by stable state.

Maximal thrust in all supply frequencies is desirable if the normal force is within the acceptable limitation. In the traditional design method, slip frequency is simply one constant below the base speed, linearly increases above the base one. Actually, maximal thrust at a given speed as a function of slip frequency can be optimized by iterative solution. The maximum thrust and relative slip frequency are shown in Fig. 6.

TABLE I

Dimensions OF THE SLIM

\begin{tabular}{|l|l|l|}
\hline Pole pitch & Pole pairs & Primary length/width \\
\hline $0.2808(\mathrm{~m})$ & 4 & $2.476 / 0.3(\mathrm{~m})$ \\
\hline Secondary width & Secondary sheet thickness & Air gap \\
\hline $0.36(\mathrm{~m})$ & $7(\mathrm{~mm})$, Copper & $9(\mathrm{~mm})$ \\
\hline Base frequency & Thrust & Primary phase voltage \\
\hline $22.5(\mathrm{~Hz})$ & $25(\mathrm{KN})$ & $635(\mathrm{~V})$ \\
\hline Line current & Number of slots & Secondary sheet width \\
\hline $280(\mathrm{~A})$ & 79 & $0.36(\mathrm{~m})$ \\
\hline
\end{tabular}

Fig. 7 shows the constant current start-up process, including the velocity, thrust, secondary fundamental-, and eddy-current curves solved by transient equations. In the overall operation region, the motor has constant stator current of $280 \mathrm{~A}$ and constant primary frequency of $10 \mathrm{~Hz}$. The secondary fundamental current, similar to that of 
RIM, decreases gradually as the speed rises, and equals zero at the synchronous point. However, the secondary end effect part ascends with its incremental speed, and reaches the maximum at the synchronous speed for the quick air gap flux linkage change resulted from the longitudinal end effect.

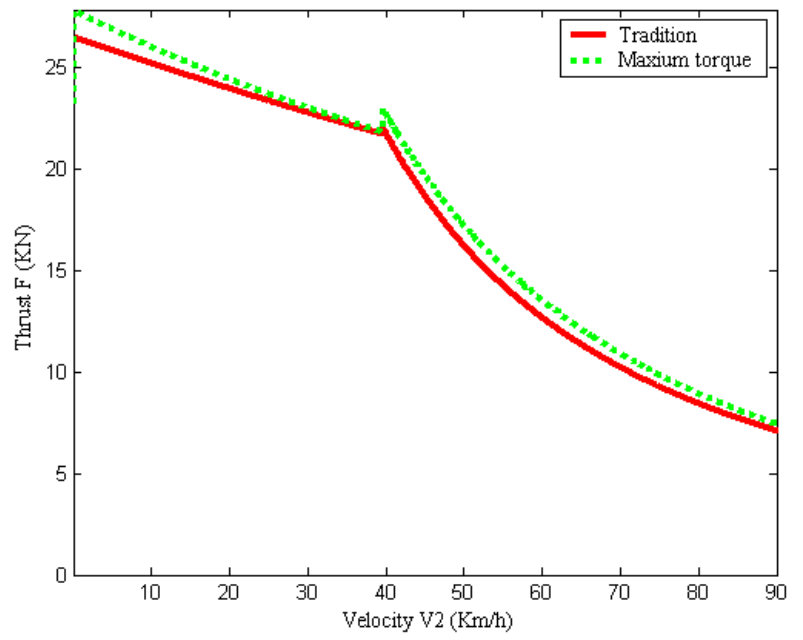

(a)

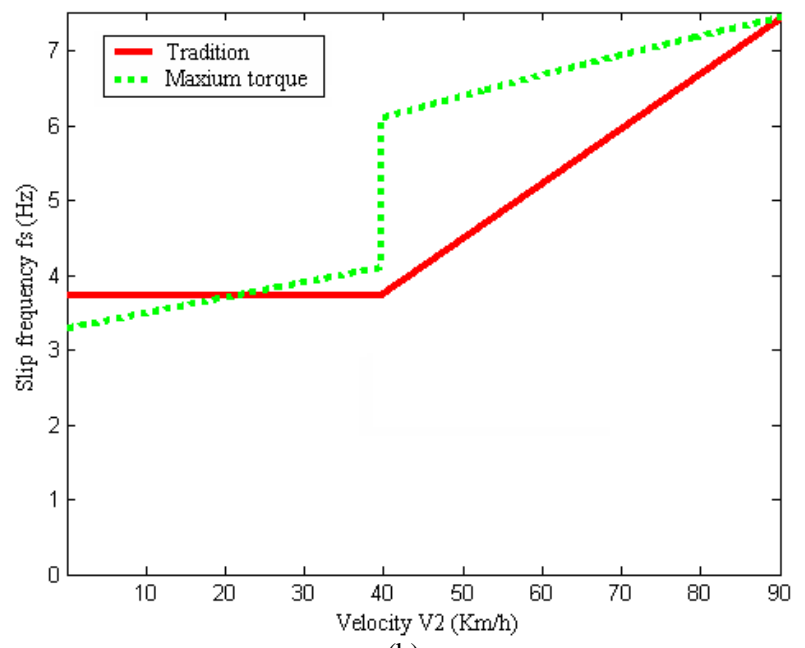

Fig. 6. Traditional slip frequency and maximal torque algorithms: (a) Torque, (b) Slip frequency.

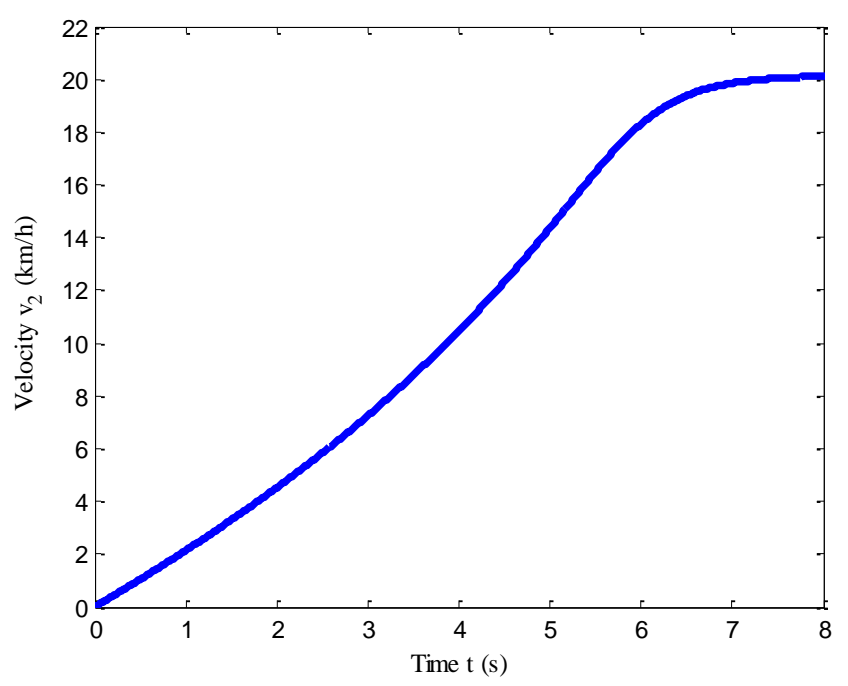

(a)

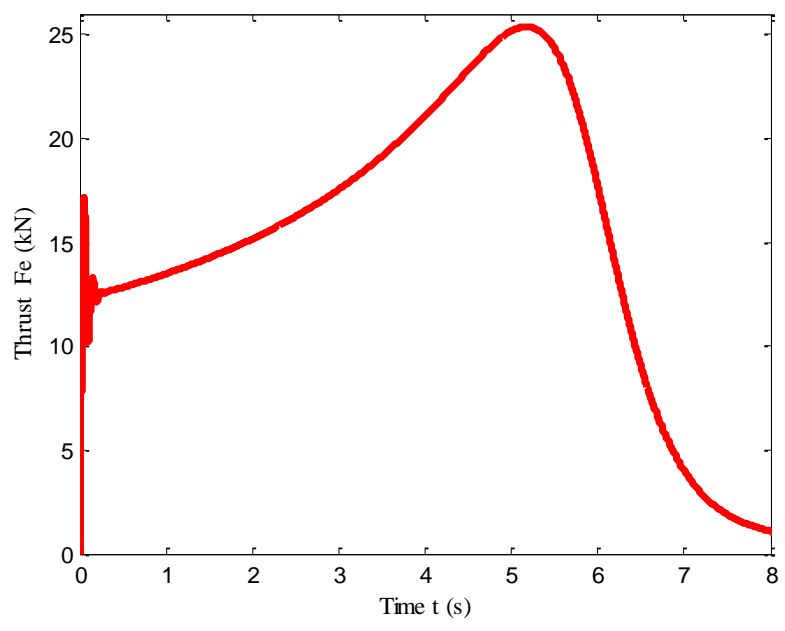

(b)

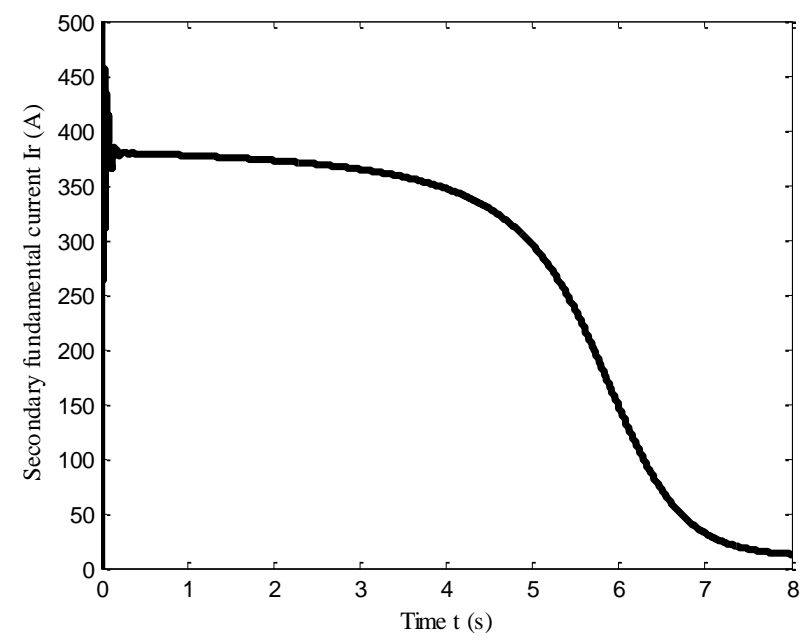

(c)

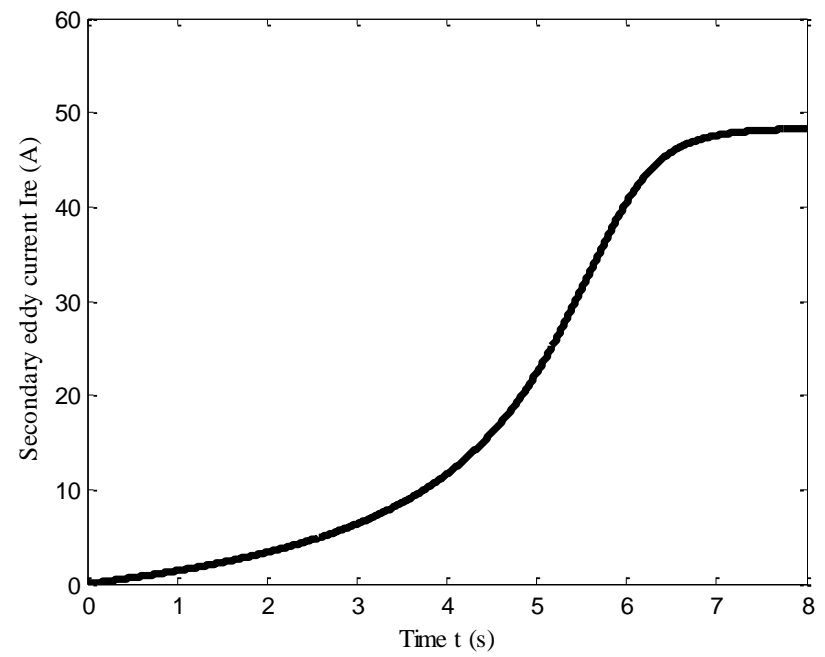

(d)

Fig. 7. Constant current start-up performance analysis: (a) velocity, (b) thrust, (c) secondary fundamental current (RMS), and (d) secondary eddy current.

\section{CONCLUSIONS}

This paper has set out an improved equivalent circuit of SLIM based on winding function method. The end effects are automatically included in machine parameters. It can analyze steady and dynamic states of SLIM in a similar way to RIM. This circuit is a very useful way to study steady, dynamic and control schemes in SLIM. 


\section{REFERENCES}

[1] X. L. Long, Theory and Magnetic Design Method of Linear Induction Motor, China: Science publishing company, 2006, pp.50-100.

[2] G. Kang and K. Nam, "Field-oriented control scheme for linea induction motor with the end effect," IEE Proceeding on Electrical Power Application, vol. 152, no. 6, pp. 1565-1572, Nov. 2005.

[3] T. Higuchi and S. Nonaka,"On the design of high efficiency linear induction motors for linear metro," Electrical Engineering in Japan, vol. 137, no. 2, pp. 36-43, Aug. 2001

[4] C. A. Lu "A new coupled-circuit model of a linear induction motor and its application to steady-state, dynamic and control studies," Thesis of Queen University, Canada, 1993, pp. 22-27.

[5] D. C. Meeker and M. J. Newman, "Indirect vector control of a redundant linear induction motor for aircraft launch," Proceedings of the IEEE, vol. 97, no. 11, pp. 1768-1776, Nov. 2009.

[6] W. Rong Jong and L. Jeng Dao, "Adaptive fuzzy-neural-network control for maglev transportation system," IEEE Trans. Neural Networks, vol. 19, no.1, pp. 54-70, Jan. 2008.

[7] W. M. Rossini, et al., "New concept for lifting in onshore oil wells," IEEE Trans. Ind. Appl., vol. 44, no. 4, pp. 951-961, Jul./Aug. 2008.

[8] W. Xu, "Research on the performance of single-sided linear induction motor," Ph.D. Dissertation, Institute of Electrical Engineering, Chinese Academy of Sciences, China, 2008.

[9] A. H. Selcuk and H. Kurum, "Investigation of end effects in linear induction motors by using the finite-element method," IEEE Trans. Magn., vol. 44, no.7, pp. 1791-1795, Jul. 2008.

[10] W. Xu, J. G. Zhu, Y. G. Guo, Y. C. Zhang, and Y. Wang, "Equivalent circuits for single-sided linear induction motors," in Proc. IEEE Energy Conversion Congress and Exposition, Sept. 2009, pp. 1288-1295.

[11] J. Duncan, "Linear induction motor-equivalent-circuit model," Electric Power Applications, IEE Proceedings B, vol. 130, no.1, pp. 51-57, Jan.1983.

Wei Xu was born in Chongqing, China, in 1980. He received the B.E., B.A., and M.E. degrees from Tianjin University, Tianjin, China, in 2002 and 2005, respectively, and the Ph.D. degree from the Institute of Electrical Engineering, Chinese Academy of Sciences, Beijing, China, in 2008, all in electrical engineering.

$\mathrm{He}$ is currently a Postdoctoral Research Fellow with the Center for Electrical Machines and Power Electronics, University of Technology Sydney (UTS), Sydney, Australia. Meanwhile, he has been invited as the adjunct associate professor in Tianjin University, Sichuan University, both in China since June 2010. His current work is supported by the Early Career Researcher Grant and partly supported by the Chancellor's
Postdoctoral Research Fellowship, both at UTS. His research interests mainly include electromagnetic design and performance analysis of linear/rotary machines, including induction, permanent magnet, switched reluctance, and other emerging novel structure machines.

Jian Guo Zhu received the B.E. degree from Jiangsu Institute of Technology, Zhenjiang, China, in 1982, the M.E. degree from Shanghai University of Technology, Shanghai, China, in 1987, and the Ph.D. degree from the University of Technology Sydney (UTS), Sydney, Australia, in 1995.

He is currently a Professor of electrical engineering and the Head of the School of Electrical, Mechanical, and Mechatronic Systems, UTS. His research interests include electromagnetics, magnetic properties of materials, electrical machines and drives, power electronics, and renewable energy systems.

Yong Chang Zhang received the B.S. degrees from Chongqing University, China, in 2004 and the Ph.D. degree from Tsinghua University, in 2009, respectively, both in electrical engineering.

He is currently a postdoctoral research fellow with the University of Technology, Sydney. His research interests include sensorless and high performance control of AC motor drives, control of multilevel converters, pulsewidth modulation, PWM rectifier, advanced digital control with real time implementation.

Youguang Guo was born in Hubei, China in 1965. He received the B.E. degree from Huazhong University of Science and Technology (HUST), China in 1985, the M.E. degree from Zhejiang University, China in 1988, and the PhD degree from University of Technology, Sydney (UTS), Australia in 2004, all in electrical engineering.

From 1988 to 1998, he lectured in the Department of Electric Power Engineering, HUST. From March 1998 to July 2008, he worked as visiting research fellow, PhD candidate, postdoctoral research fellow and research fellow in the Center for Electrical Machines and Power Electronics, Faculty of Engineering, UTS. He is currently a lecturer at the School of Electrical, Mechanical and Mechatronic Systems, UTS.

His research fields include measurement and modeling of magnetic properties of magnetic materials, numerical analysis of electromagnetic field, electrical machine design and optimization, power electronic drives and control. In these fields, he has published over 230 refereed technical papers including 110 journal articles.

Guang Yong Sun was born in Chongqing, China, in 1981. He received the B.E., from Hunan University, Changsha, China, in 2003 and expects to receive his Ph.D. degree from Hunan University in 2010.

$\mathrm{He}$ is currently a visiting student in the School of Aerospace, Mechanical and Mechatronic Engineering, University of Sydney, Sydney, Australia. His research interests include sheet metal forming, structure optimization and automotive safety. 